Gefässchirurgie 2014 · 19:338-338

DOI 10.1007/s00772-014-1358-2

Online publiziert: 10. Juli 2014

๑) Springer-Verlag Berlin Heidelberg 2014

K.M. Balzer ${ }^{1}$ - N. Diehm ${ }^{2}$ H.-H. Eckstein ${ }^{3}$

${ }^{1}$ Klinik für Gefäß- und Endovaskularchirurgie, St. Marien Hospital Bonn

2 Schweizer Herz- und Gefässzentrum Bern, Universitätsklinik für Angiologie, Inselspital, Bern

${ }^{3}$ Klinik und Poliklinik für Vaskuläre und Endovaskuläre Chirurgie, Klinikum

rechts der Isar der Technischen Universität München

\title{
Vaskuläre Leitlinien
}

anderweitig für die jeweilige Thematik in besonderer Weise ausgewiesen sind.

Wir sind sehr froh, mit Herrn Prof. Dr. Peter Ringleb aus der Neurologischen Universitätsklinik Heidelberg einen national und international sehr geschätzten Experten aus dem Bereich der vaskulären Neurologie gefunden zu haben, der für dieses Heft der Gefässchirurgie die von der Deutschen Gesellschaft für Allgemeinmedizin und Familienmedizin (DEGAM) im Jahr 2012 herausgegebenen Leitlinie „Schlaganfall“ (AWMF-Register-Nr. 053-011) in hervorragender Weise zusammengefasst hat. In weiteren Heften der Gefässchirurgie werden aktuelle Leitlinien u. a. zur Antikoagulation, zur arteriellen Hypertonie und zur Nierenarterienstenose behandelt werden.

Wir hoffen sehr, dass unsere neue Rubrik „Vaskuläre Leitlinien“ positiv aufgenommen wird und freuen uns auf konstruktive Vorschläge und Rückmeldungen.

\section{Mit besten Grüßen}

Ihre

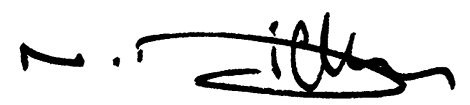

Prof. Dr. N. Diehm

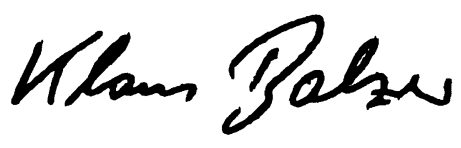

Priv.-Doz. Dr. K.M. Balzer

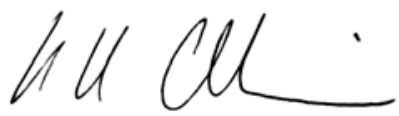

Prof. Dr. H.-H. Eckstein

\section{Korrespondenzadresse

PD Dr. K.M. Balzer
Klinik für Gefäß- und Endo-
vaskularchirurgie, St. Marien
Hospital Bonn
Robert-Koch-Str. 1,53115 Bonn
kai.balzer@
marien-hospital-bonn.de

Prof. Dr. N. Diehm
Schweizer Herz- und Gefäss-
zentrum Bern, Universitätskli-
nik für Angiologie, Inselspital
3010 Bern
Schweiz
nicolas.a.diehm@gmail.com

Prof. Dr. H.-H. Eckstein
Klinik und Poliklinik für Vasku-
läre und Endovaskuläre Chir-
urgie, Klinikum rechts der Isar
der Technischen Universität
München
Ismaninger Str. 22,
81675 München
München
hheckstein@web.de

Interessenkonflikt. Die korrespondierenden Autoren geben an, dass kein Interessenkonflikt besteht. 